OPEN ACCESS

Edited by:

Yiqi Zhang,

Xi'an Jiaotong University, China

Reviewed by:

Xiao-Dong Chen,

Sun Yat-sen University, China

Chen Shen,

Rowan University, United States

Fangwei Ye,

Shanghai Jiao Tong University, China

${ }^{*}$ Correspondence:

Cuicui LU

cuicuilu@bit.edu.cn

Specialty section:

This article was submitted to

Optics and Photonics,

a section of the journal

Frontiers in Physics

Received: 08 December 2021

Accepted: 10 January 2022

Published: 03 February 2022

Citation:

Elshahat S and Lu C (2022) Bidirectional Rainbow Trapping in 1-D Chirped Topological Photonic Crystal.

Front. Phys. 10:831203.

doi: 10.3389/fphy.2022.831203

\section{Bidirectional Rainbow Trapping in 1-D Chirped Topological Photonic Crystal}

\author{
Sayed Elshahat ${ }^{1,2}$ and Cuicui Lu ${ }^{1,3 *}$ \\ ${ }^{1}$ Key Laboratory of Advanced Optoelectronic Quantum Architecture and Measurements of Ministry of Education, Beijing Key \\ Laboratory of Nanophotonics and Ultrafine Optoelectronic Systems, School of Physics, Beijing Institute of Technology, Beijing, \\ China, ${ }^{2}$ Physics Department, Faculty of Science, Assiut University, Assiut, Egypt, ${ }^{3}$ Collaborative Innovation Center of Light \\ Manipulations and Applications, Shandong Normal University, Jinan, China
}

The rainbow trapping effect has attracted gathering attention due to its potential application in data processing, energy storage, and light-matter interaction enhancement. The interest has increased recently with the advent of topological photonic crystals (PCs), as the topological PC affords a robust platform for nanophotonic devices. We proposed a chirped one-dimensional (1D) PC as a sandwiched trapped between two1D topological PCs to realize two topological edge states (TESs) for topological protection and trap the formed rainbow. Through graded the thickness of dielectric layers of the chirped 1D PC, light of different wavelengths components localizes and stores at different spatial positions leading to rainbow trapping formation. Unidirectional rainbow trapping can be observed by progressively increasing the thicknesses of the chirped PC. Nonetheless, changing increasingly one of its thicknesses and solidifying the other leads to bidirectional rainbow trapping. Achieving bidirectional rainbow trapping will reduce the footprint of nanophotonic devices in the future. This work brings inspiration to the realization of the rainbow trapping effect and provides a way to design topological nanophotonic devices.

\footnotetext{
Keywords: rainbow trapping, topological photonic crystal, nanophotonic devices, chirped photonic crystal, topological edge state
}

\section{INTRODUCTION}

Photonics is principally concerned with the wave properties of frequency, wavevector, and polarization, representing the degrees of freedom of essential information for any optical system. Frequency plays an instrumental part in integrated photonic devices. In particular, it has been shown that in a tapered metamaterial structure, light can be trapped and slowed down in exact positions depending on its frequency [1]. This phenomenon is named a trapped rainbow, just as sunlight is scattered in a continuous color spectrum through a prism (hence the name is rainbow). The effect can also be considered as the spatial separation of the frequency components of the propagating wave. The appearance of rainbow trapping offers a novel technique for frequency routing of slow light [1]. After the formation of the first theoretical work, many successive methods were presented to realize rainbow trapping, such as metamaterials [1,2], metasurfaces [3], plasmonic structures [4-6], phononic crystals in one-dimensional (1D) [7] and two-dimensional (2D) [8], and photonic crystals (PCs), in 1D [9], 2D [10, 11] and three-dimensional (3D) [12]. Most of the mentioned methods depend on either metallic or dielectric materials. However, metallic materials are lossy at optical wavelengths. Thus, the fully dielectric structure (PCs) is an excellent alternative to realizing rainbow trapping. 


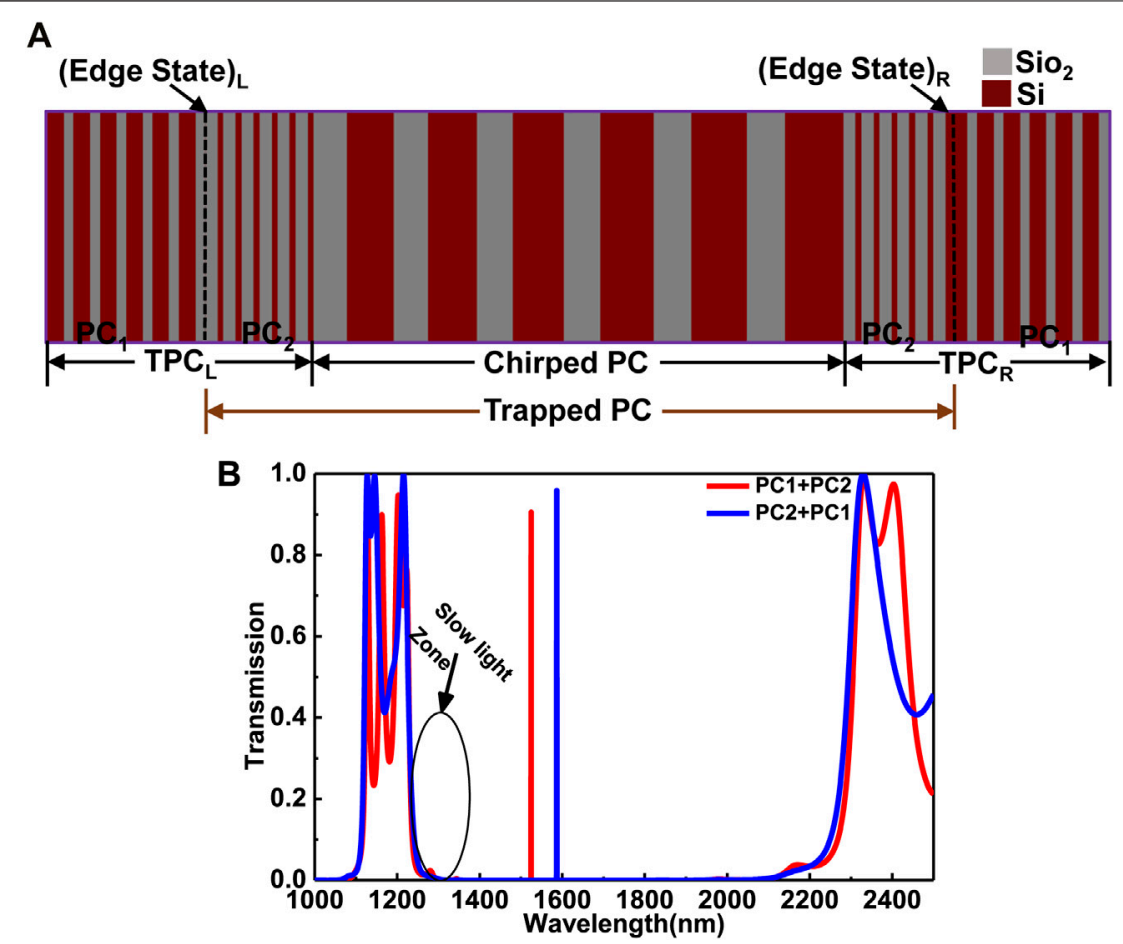

FIGURE 1 | (A) Schematic diagram of 1D PC heterostructure; (B) the transmission spectrum of $\mathrm{TPC}_{\mathrm{L}}$ andTPC $\mathrm{R}_{R}$.

The rainbow trapping effect has attracted attention due to its potential applications in data processing, energy storage, and light-matter interaction enhancement. The interest has increased recently with the advent of topological PCs, which affords a robust platform for optical devices. Consequently, the combination of topology and rainbow can make possible new potentials designing topologically protected photonic devices. Due to the complex design and manufacturing structure of 2D and 3D PCs, topological PCs are preferred in the 1D structure for the advantages of simple design and ease of fabrication.

In this paper, the proposed structure is based on two-1D topological PCs to realize two topological edge states (TESs) for topological protection and trap the formed rainbow. A chirped 1D PC is inserted as a sandwiched between the two-1D topological PCs. The way to achieve the rainbow is to set the structure (or chirp) statically through one or more basic structural parameters such as position, size, and refractive index that gradually change along the direction of propagation. Through graded the thickness of dielectric layers of the chirped 1D PC, light of different wavelengths components localizes and stores at different spatial positions leading to rainbow trapping formation. The interesting observation is bidirectional rainbow trapping, increasing the thickness of one layer kind progressively and solidifying a different kind. Thus, the light propagates and localizes in both directions in the trapped area between the two TESs. Then, different frequencies can be segregated at different positions in both directions. To the best of our knowledge, this type of rainbow trapping has not been observed in topological photonics. Achieving bidirectional rainbow trapping will reduce the footprint of the nanophotonic device in the future. It is possible for this type of rainbow trapping to have numerous applications, for example, a bidirectional optical filter, a bidirectional laser, etc.

\section{Designs and Results}

The topological heterostructure of $\mathrm{PC}$ is based on two topological PCs, namely $\mathrm{TPC}_{\mathrm{L}}$ and $\mathrm{TPC}_{\mathrm{R}}$ as shown in Figure 1A. TPC $\mathrm{L}$ is composed of two PCs $\left(\mathrm{PC}_{1}\right.$ and $\left.\mathrm{PC}_{2}\right)$, from the same dielectric materials of silicon $\mathrm{Si}$ and silicon dioxide $\mathrm{SiO}_{2}$ with refractive indices $n_{S i}=3.48$ and $n_{\mathrm{SiO}_{2}}=1.45$, respectively. $\mathrm{TPC}_{\mathrm{R}}$, is composed of two PCs $\left(\mathrm{PC}_{2}\right.$ and $\left.\mathrm{PC}_{1}\right)$, is the mirror image of $\mathrm{TPC}_{\mathrm{L}} \cdot \mathrm{PC}_{1}$ consists of six consecutive layers from $\mathrm{Si}$ and $\mathrm{SiO}_{2}$ of layer thicknesses $d_{1 S i}=350 \mathrm{~nm}$, and $d_{1 S i O_{2}}=225 \mathrm{~nm}$, respectively, and the lattice constant of $\mathrm{PC}_{1}$ is $a_{1}=575 \mathrm{~nm}$. The thicknesses of $\mathrm{PC}_{2}$, are evaluated based on [13], are $d_{2 \mathrm{SiO}_{2}}=$ $276 \mathrm{~nm}$ and $d_{2 S i}=115 \mathrm{~nm}$ and consists of six consecutive layers from $\mathrm{SiO}_{2}$ and $\mathrm{Si}$, respectively with $a_{2}=391 \mathrm{~nm}$. The TESs can exist in $\mathrm{TPC}_{\mathrm{L}}\left(\mathrm{PC}_{1}+\mathrm{PC}_{2}\right)$ and $\mathrm{TPC}_{\mathrm{R}}\left(\mathrm{PC}_{2}+\mathrm{PC}_{1}\right)$ at the heterostructure interface because the two $\mathrm{PCs}$ possess bandgaps in the same wavelength range with different topological properties [14]. The transmission spectrum of $\mathrm{TPC}_{\mathrm{L}}$ and $\mathrm{TPC}_{\mathrm{R}}$ is shown in Figure 1B using the finite element method (FEM), solver package of COMSOL Multiphysics, with perfect matched layer boundary condition (PML). Two TESs formed at the left and right interfaces with a high resonated transmission peak between the two stacked PCs. The two TESs areas appeared inside the bandgap, allowing two areas of slow light zones near the bandgap edges. Whereas the two edge states work as a topological cavity to trap the light inside the 

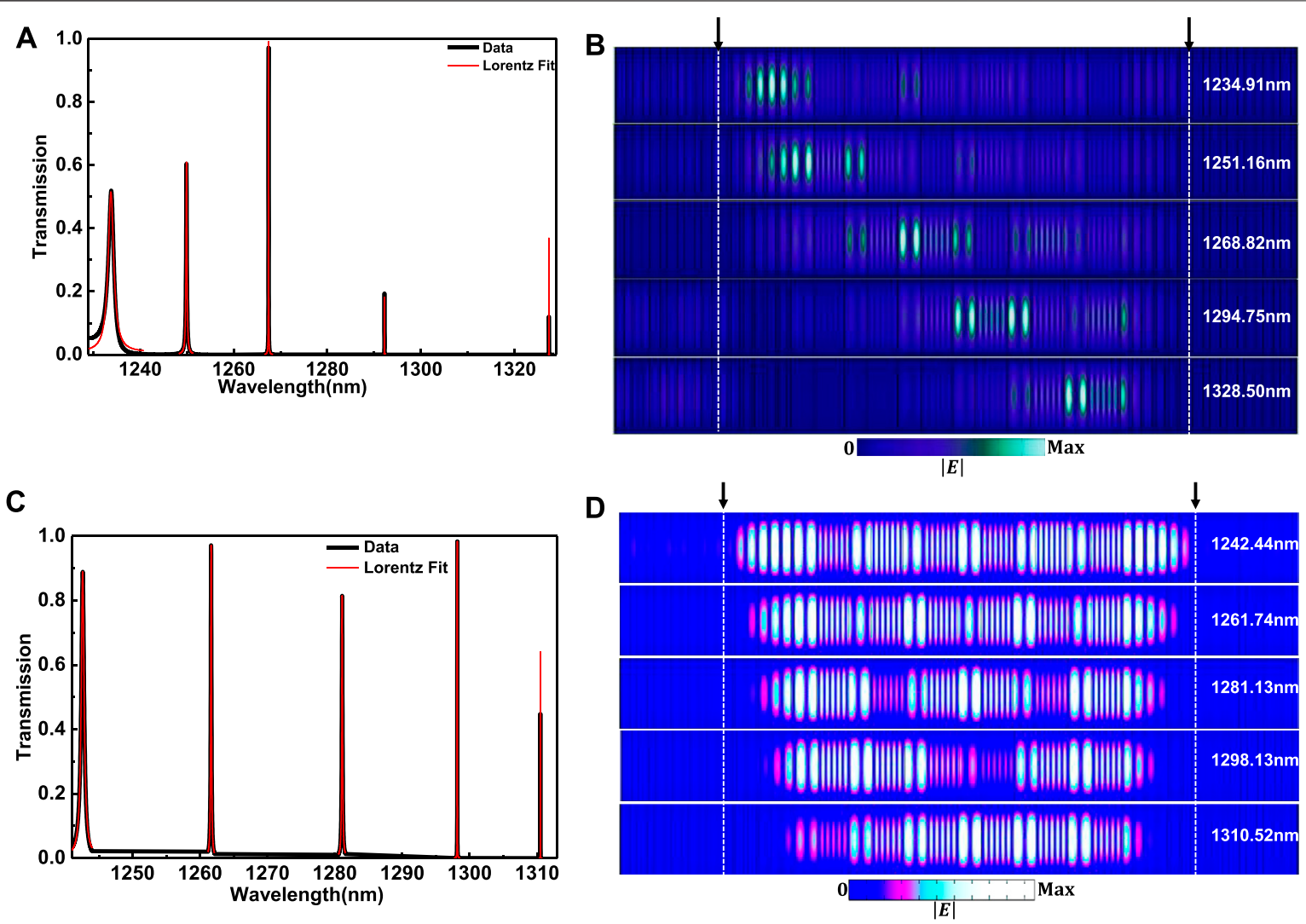

FIGURE 2 | (A) The transmission spectrum of the unidirectional rainbow trapping structure with the Lorentzian-fitting curves when $d_{\mathrm{SiO}_{2}}$ is varying from 740 to $765 \mathrm{~nm}$ and $d_{S i}$ from 1,000 to $1,100 \mathrm{~nm}$; (B) the electric field $|\mathrm{E}|$ intensity distribution of the unidirectional rainbow trapping at wavelengths

1234.91, 1251.16, 1268.82, 1294.75, and 1328.50 by nm; (C) the transmission spectrum of the bidirectional rainbow trapping structure with the Lorentzian-fitting curves of $d_{\mathrm{SiO}_{2}}$ varying from 740 to $765 \mathrm{~nm}$ and $d_{\mathrm{Si}}$ is fixed at $1,060 \mathrm{~nm}$; (D) the |E| intensity distribution of the bidirectional rainbow trapping at wavelengths $1242.44,1261.74,1281.13,1298.13$, and 1310.52 by $\mathrm{nm}$

chirped structure, which may enhance the field localization and quality factor.

A linearly chirped 1D PC is inserted as a sandwiched between the two-1D topological PCs. The chirped 1D PC is composed of six consecutive layers from $\mathrm{SiO}_{2}$ and $\mathrm{Si}$. It started from $\mathrm{SiO}_{2}$ to avoid the edge states between $\mathrm{PC}_{2}$ and the chirped one. Consequently, $\mathrm{PC}_{2}$, chirped $\mathrm{PC}$ and $\mathrm{PC}_{2}$ formed a trapped $\mathrm{PC}$, and the formed rainbow trapping will be topologically protected by the two TESs as shown in Figure 1A. The possibility for the light entering the trapped PC to escape is slight because the two edge states work like topological cavities. The thicknesses of $\mathrm{SiO}_{2}$ and $\mathrm{Si}$ layers are $d_{\mathrm{SiO}_{2}}$ and $d_{\mathrm{Si}}$ respectively. Through graded $d_{\mathrm{SiO}_{2}}$ linearly from 740 to $765 \mathrm{~nm}$ and $d_{S i}$ from $1,000 \mathrm{~nm}$ to $1,100 \mathrm{~nm}$, the light can be trapped and slowed down in exact position depending on its frequency, forming a rainbow trapping effect.

\section{Unidirectional and Bidirectional Rainbow Trapping}

As mentioned above, achieving rainbow trapping is to set the structure statically through one or more basic structural parameters that gradually change along the propagation direction. Unidirectional rainbow trapping can be observed by gradually increasing $d_{\mathrm{SiO}_{2}}$ and $d_{\mathrm{Si}}$ of the chirped PC sandwiched between the two topological PCs. $d_{\mathrm{SiO}_{2}}$ is varying from 740 to $765 \mathrm{~nm}$ with an increment of $5 \mathrm{~nm}$ and $d_{S i}$ from 1,000 to $1,100 \mathrm{~nm}$ with an increment of $20 \mathrm{~nm}$. Figure $\mathbf{2 A}$ shows the transmission spectrum of the proposed structure with the fitting curves, which are symmetric in the representative Lorentzian-line shape. The resonated transmission peaks at $\mathrm{nm}$ wavelengths are $1234.91,1251.16,1268.82,1294.75$, and 1328.50, with the full width at half maximum (FWHM) by $\mathrm{nm}$ is $1.41444,0.23029,0.05598,0.01136$, and $5.86 \times 10^{-4}$ respectively. The quality factor $(Q)$, which is the ratio of the resonant wavelength to FWHM, at each resonated transmission peak is $873.07,5.43 \times 10^{3}, 2.26 \times 10^{4}, 1.13 \times 10^{5}$, and $2.26 \times 10^{6}$ respectively. The high $Q$-factor due to the robust field localization between the two TES, which led to robust optical localization in the chirped area.

To show the formed unidirectional rainbow trapping, the electric field $|E|$ intensity distribution is calculated as shown in Figure 2B. Consequently, multi-modes are excited at the desired 
wavelengths due to coupling light and the chirped structure. The $|E|$ intensity distributions point out the propagation of light inside the chirped PC. The $|E|$ intensity rises at the localization points. With gradually increasing the thicknesses of the chirped PC in the propagation direction and light propagates from left to right. In principle, the light will gradually slow down and eventually approach the "stop". The light of different wavelengths localizes at different spatial positions leading to rainbow trapping in the slow light zone near the left bandgap edge. The property of the bandgap rises when the wavelength of the incident light is in the order of the structure periodicity [15]. The left/right side of the chirped PC is composed of smaller $\left(740 \mathrm{~nm}\left(d_{\mathrm{SiO}_{2}}\right)+1000 \mathrm{~nm}\left(d_{\mathrm{Si}}\right)\right.$ /larger $\left(765 n m\left(d_{\mathrm{SiO}_{2}}\right)+1100 \mathrm{~nm}\left(d_{\mathrm{Si}}\right)\right.$ periodicity. This, in turn, affects the shorter/longer wavelengths to be trapped in the appropriate positions. As with increasing the wavelength, the trapped light moves to the right side. The lowest wavelength of $1,234.91 \mathrm{~nm}$ is localized near the left TES at the smaller periodicity. With increasing the wavelength, the $|E|$ intensity distributions move to the right till the right TES at the wavelength of $1,328.50 \mathrm{~nm}$. The rainbow trapping bandwidth changed from 1,234.91 to $1,328.50 \mathrm{~nm}$. We find that the formed rainbow is trapped between the two TESs, which act as a strong cavity and enhance the field localization and $Q$-factor.

The work can extend to construct bidirectional rainbow trapping by progressively increasing one of its thicknesses and solidifying the other. $d_{\mathrm{SiO}_{2}}$ is varying from 740 to $765 \mathrm{~nm}$ with an increment of $5 \mathrm{~nm}$ and $d_{S i}$ can be fixed at any value from 1,000 to $1,100 \mathrm{~nm}$. The results of fixing $d_{S i}$ at $1060 \mathrm{~nm}$ is only shown. Figure 2C shows the transmission spectrum of the bidirectional rainbow trapping structure with the Lorentzian-fitting curves. The resonated transmission peaks that appear at wavelengths by $\mathrm{nm}$ are $1242.44,1261.74,1281.13,1298.13$, and 1310.52 with FWHM by $\mathrm{nm}$ is $0.50719,0.13104,0.12927,0.01949$, and 0.00327 , respectively. The $Q$-factor at each resonated transmission peak is $2.45 \times 10^{3}, 9.77 \times 10^{3}, 9.76 \times 10^{3}, 6.66 \times 10^{4}$, and $4.00 \times 10^{5}$, respectively. The $|E|$ intensity distribution is shown in Figure 2D. The field localization is observed in both directions near the two TESs with a smaller wavelength. The two TESs work as a cavity to trap the light inside the chirped PC and make the possibility of light escaping slightly. With increasing the wavelength value, filed localization is shrinking into the chirped PC to form a unidirectional rainbow in both directions simultaneously. Accordingly, the different wavelengths from the wave packet can be segregated spatially at different positions in both directions, and bidirectional rainbow trapping is realized clearly. To the best of our knowledge, this kind of rainbow has not been observed yet in topological photonics. It may possess numerous applications, such as bidirectional devices. Realizing bidirectional rainbow trapping will diminish the footprint of nanophotonic devices in the future.

With increasing the number of alternative layers of the chirped PC, a higher Q-factor and multi-localized modes are obtained. Unidirectional and bidirectional rainbow trapping can be realized by considering ten periods of the chirped PC sandwiched between the two topological PCs. Through the same manner, unidirectional rainbow trapping is detected by gradually increasing $d_{\mathrm{SiO}_{2}}$ and $d_{S i}$ of the chirped PC. $d_{\mathrm{SiO}_{2}}$ is varying from 740 to $785 \mathrm{~nm}$ with an increment of $5 \mathrm{~nm}$ and $d_{S i}$ from 1,000 to $1,180 \mathrm{~nm}$ with an increment of $20 \mathrm{~nm}$. Multi-modes are increased and excited at the desired wavelengths due to coupling light and the increased chirped PC. Figure 3A shows only three localized modes of the $|\mathrm{E}|$ intensity distribution of the formed unidirectional rainbow at 1233.58, 1305.83, and 1410.54 by nm. Different wavelengths localize in different spatial locations. Whereas the wavelength increases, the locations where the light is trapped move to the right side, as shown in Figure $\mathbf{3 A}$. For the bidirectional rainbow trapping, it can be created by gradually thickening $d_{\mathrm{SiO}_{2}}$ and anchoring $d_{\mathrm{Si}}$. A bidirectional rainbow is observed for fixing $d_{S i}$ at any value from 1,000 to $1,180 \mathrm{~nm}$ with $d_{\mathrm{SiO}_{2}}$ is varying from $740 \mathrm{~nm}$ to $785 \mathrm{~nm}$. Figure 3B shows the results of fixing $d_{S i}$ at $1060 \mathrm{~nm}$ of the $|\mathrm{E}|$ intensity distribution of the bidirectional rainbow trapping at 1239.93, 1277.23, and 1308.64 by $\mathrm{nm}$. It seen clearly from Figure $\mathbf{3 B}$, as the wavelength value increases, the localization deposited in the chirped PC shrinks to form a unidirectional rainbow in both directions simultaneously. Hence, the bidirectional rainbow can be achieved. Summing up, as the number of periods increases, the transmittance remains high due to both TESs serving as a light-trapping cavity within the chirped PC, but with a very fine meshing and a small step size. It should be noted that increasing the number of periods will increase the manufacturing effort and cost. The less of the periods, the smaller size of the structures, and it is better for the integration of nanophotonic devices. We use six smaller periods for both topological PCs and chirped PC; thus, the trapped PC is 18 periods under the premise of good performance.

\section{FDTD Validation}

The proposed PC heterostructure was modeled by the finitedifference-time-domain (FDTD) method to validate the propagation properties. A high resolution (grid size for each dimension) of $30 / \boldsymbol{a}_{1}$ is used, which is small enough to resolve a minor feature in the fields and structure during the simulation. A PML boundary is added to the edge of the domain of about $\boldsymbol{a}_{1}$ to absorb all incident energy without producing reflections. A Gaussian source was launched for FDTD calculation and placed in the middle of the trapped PC, as shown in Figure 4A. During the simulation, two monitors are introduced to study the spectral characteristics of the structure at specific wavelength/frequency values and used to measure the steady-state properties for the field distribution. The first and second monitors (M1 and M2) are placed at the trapped PC's beginning and ending, respectively. Figures $4 B, C$ show the electric field intensity amplitude $\left(\boldsymbol{E}_{z}^{2}\right)$ along the propagation $(\mathrm{z})$ of unidirectional and bidirectional rainbow trapping, respectively, at the center point of the $x$ - $y$-plane.

In the case of unidirectional rainbow trapping of $\boldsymbol{d}_{\mathrm{SiO}_{2}}$ linearly varies from 740 to $765 \mathrm{~nm}$ with $\boldsymbol{d}_{S i}$ from 1,000 to $1,100 \mathrm{~nm}$, and based on the above explanation, at low wavelengths, M1 records the highest amplitude of the electric field intensity indicating the field localization, and the amplitude is minimized at M2 simultaneously. When the wavelength increases, the intensity begins to decrease at M1 
A

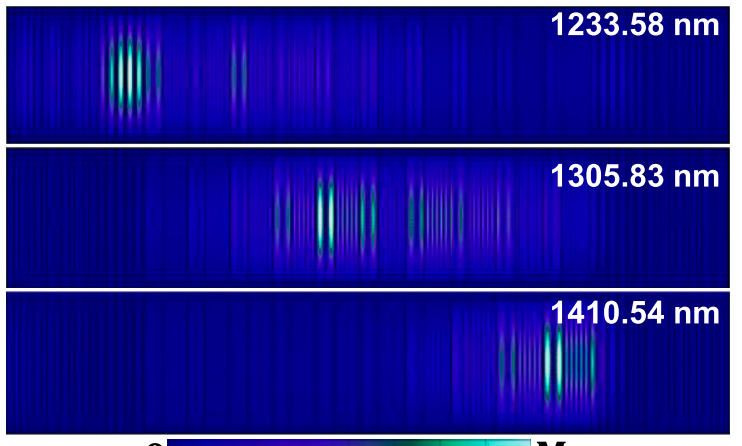

0

$|E|$

\section{B}

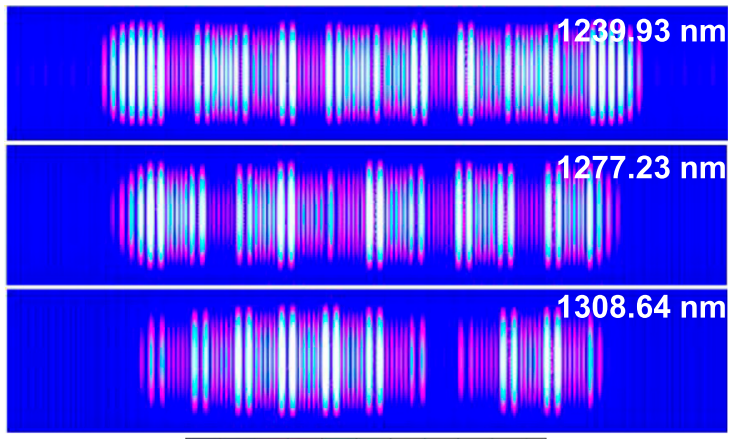

0

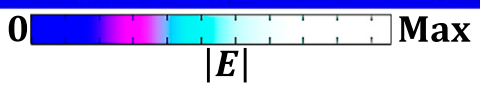

FIGURE 3 | (A) The |E| intensity distribution of the unidirectional rainbow trapping at wavelengths $1233.58,1305.83$, and 1410.54 by $\mathrm{nm}$ when $d_{S \mathrm{O}_{2}}$ is varying from $740 \mathrm{~nm}$ to $785 \mathrm{~nm}$ with an increment of $5 \mathrm{~nm}$ and $d_{S i}$ from $1000 \mathrm{~nm}$ to $1180 \mathrm{~nm}$ with an increment of $20 \mathrm{~nm}$; (B) the $\mid$ E| intensity distribution of the bidirectional rainbow trapping at wavelengths $1239.93,1277.23$, and 1308.64 by $\mathrm{nm}$, when $d_{\mathrm{SiO}_{2}}$ is varying from $740 \mathrm{~nm}$ to $785 \mathrm{~nm}$ with an increment of $5 \mathrm{~nm}$ and $d_{S i}$ is fixed at $1060 \mathrm{~nm}$.

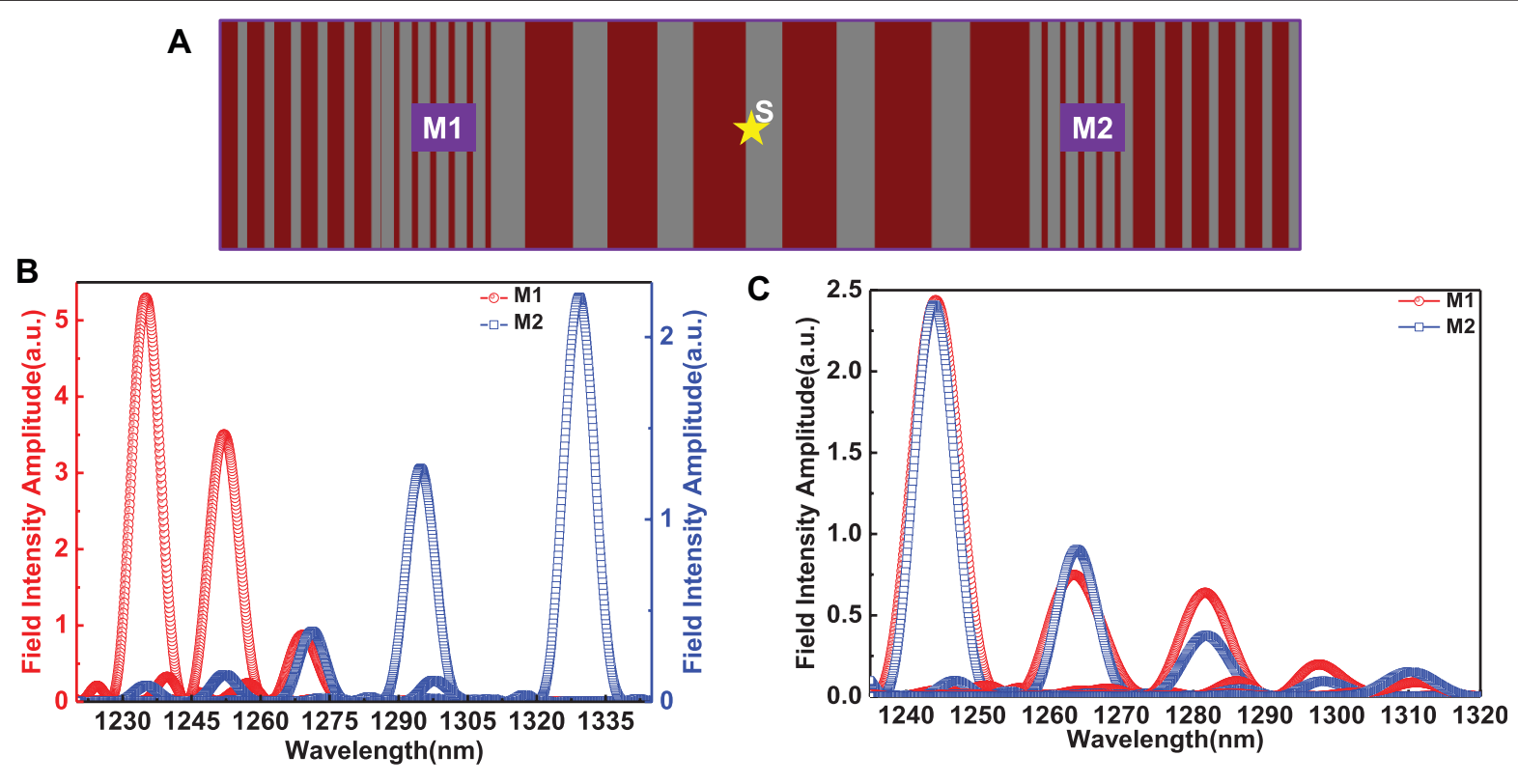

FIGURE 4 | (A) FDTD set up of grid size 30/a w with placing a Gaussian source in the middle of the trapped PC and two monitors (M1, M2) to the left and right, respectively; The electric field intensity amplitude $\left(E_{z}^{2}\right)$ of $\mathbf{( B )}$ unidirectional $\mathbf{( C )}$ bidirectional rainbow trapping.

gradually and vice versa at M2 because the field localization moves progressively from left to right with increasing the wavelength, which is illustrated in Figure 4B. A complete agreement with the previous section's results as shown in Figure 2B. However, there is a minor disparity in the values of wavelengths between FEM and FDTD methods, principally originating from the limited discretization of grid size in FDTD and the number of mesh points for FEM calculations.

Then, to verify bidirectional rainbow trapping, the same above condition are fixed but with $d_{\mathrm{SiO}_{2}}$ is varying from 740 to $765 \mathrm{~nm}$ and $d_{S i}$ is fixed at $1,060 \mathrm{~nm}$. The highest field intensity of M1 is located sequentially in the trapped PC from the left side (with the smallest wavelength) to the right side (with the largest wavelength). On the contrary, for M2, the highest field intensity is located sequentially in the trapped PC from the right side (with the smallest wavelength) to the left side (with the largest wavelength), as shown in Figure 4C, confirming the results in Figure 2D. The two TESs works as a cavity which cases exchange with transfer the energy flow between the two cavities of the forward and backward propagation. Meanwhile, rainbow 


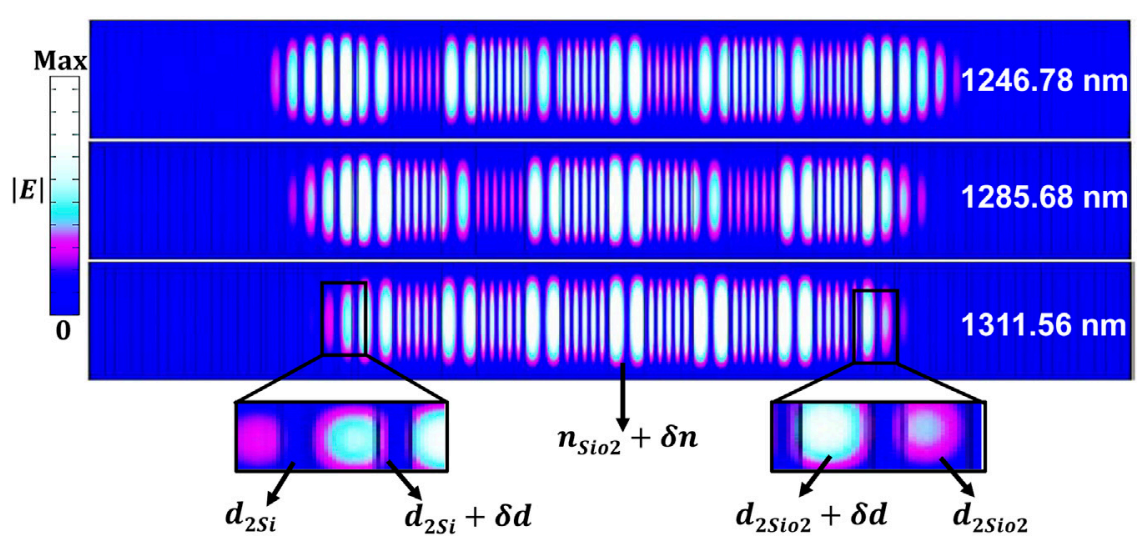

FIGURE 5 |Bidirectional rainbow trapping realization with disordering of some parameters, the inset shows the deformation in last and first layers of $\mathrm{PC} \mathrm{C}_{2}$ from $\mathrm{TPC}_{\mathrm{L}}$ and $\mathrm{TPC}_{\mathrm{R}}$ respectively.
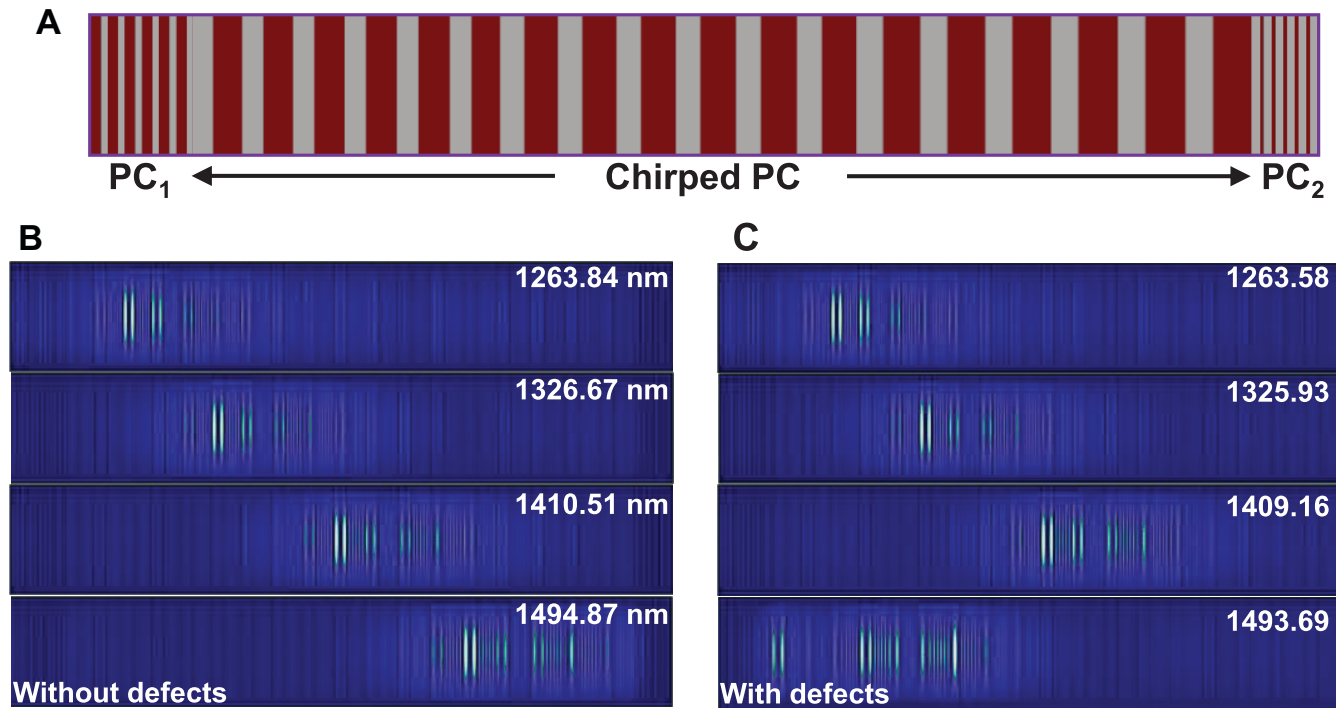

C

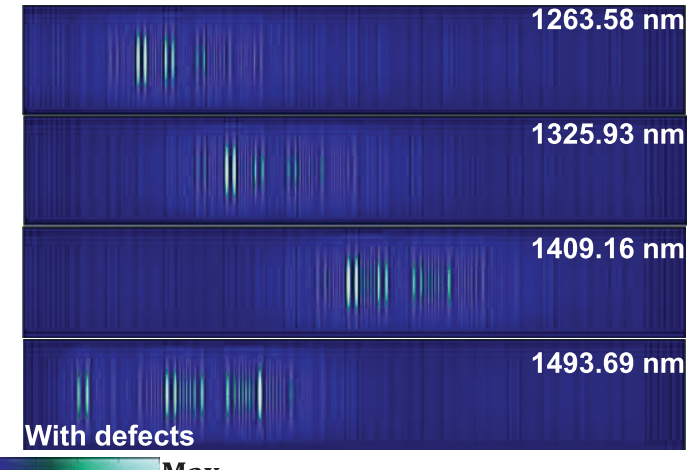

$|\boldsymbol{E}|$

FIGURE 6 | (A) Schematic diagram of the chirped PC sandwiched between two PCs. The |E| intensity distribution of the trivial unidirectional rainbow trapping of 18 periods of the trapped chirped $\mathrm{PC}$ between $\mathrm{PC}_{1}$ and $\mathrm{PC}_{2} \mathbf{( B )}$ without defects; $(\mathbf{C})$ with defects by the disordering of some parameters (deformation in last and first layers of $\mathrm{PC}_{1}$ and $\mathrm{PC}_{2}$ respectively).

trapping is observed in both FEM and FDTD methods, which confirms our simulation results.

\section{Immune to Defects}

The critical step is to confirm that the formed rainbow is robust and topologically protected from disorders by the two TESs on both sides. Some disorders are introduced in the trapped PC $\left(\mathrm{PC}_{2}\right.$, chirped $\mathrm{PC}$ and $\left.\mathrm{PC}_{2}\right)$ between the two TESs through changing thickness or refractive index of some layers. Three cases are considered; the last layer of $\mathrm{PC}_{2}\left(d_{2 S i}\right)$ from the $\mathrm{TPC}_{\mathrm{L}}$ is deformed by $\delta d= \pm 0.05 a_{2}$. Via the same amount of deformation $\left( \pm 0.05 a_{2}\right)$ is applied on the first layer of $\mathrm{PC}_{2}$ $\left(d_{2 S i}\right)$ from the TPC $C_{R}$. The last case is changing the refractive index of the middle $\mathrm{SiO}_{2}$ in the chirped PC by $\delta n= \pm 0.01$. By applying each case individually or combined, rainbow tapping is achieved with a slight shifting in the wavelength. Figure 5 shows the realization of bidirectional rainbow trapping with disordering of all deformed cases together. We can see clearly, the $|E|$ intensity distribution is highly localized in the trapped PC due to topological protection by the two TESs even in the presence of disorders. Bidirectional rainbow trapping is still observed on both sides but with slight shifting in 
wavelengths. Accordingly, the formed rainbow is robust and immune to disorders.

\section{Trivial Rainbow Trapping}

Trivial rainbow trapping is realized through the same modality by introducing a chirped PC as a trapped structure between two conventional PCs. In the exact mechanism as mentioned above, introducing a chirped PC as a sandwiched between two topological PCs $\left(\mathrm{TPC}_{\mathrm{L}}\right.$ and $\left.\mathrm{TPC}_{\mathrm{R}}\right)$. Under the same conditions and variables, values of $\mathrm{PC}_{1}$ and $\mathrm{PC}_{2}$ are setups, and the chirped PC consists of 18 periods from $\mathrm{SiO}_{2}$ and $\mathrm{Si}$ as shown in Figure 6A. The bidirectional trivial rainbow trapping as in the content of the topological case cannot be formed when $d_{S i}$ is fixed at any value from 1,000 to $1,340 \mathrm{~nm}$ and $d_{\mathrm{SiO}_{2}}$ is varying from 740 to $825 \mathrm{~nm}$. This is because the two TESs act as a cavity to transfer the energy flow between the two cavities of the forward and backward propagation and make the possibility of light escaping slightly. Therefore, the formed bidirectional rainbow may occur in the topological case.

Unidirectional trivial rainbow trapping can be formed with the same conditions for topological cases. When $d_{\mathrm{SiO}_{2}}$ is varying from 740 to $825 \mathrm{~nm}$ with an increment of $5 \mathrm{~nm}$ and $d_{S i}$ from 1,000 to $1,340 \mathrm{~nm}$ with an increment of $20 \mathrm{~nm}$, as shown in Figure 6B. The trivial unidirectional rainbow trapping is observed but lacks highly field localization and light confinement compared with the topological case due to the robust field localization between the two TES, which resulted in a robust optical localization in the chirped region. Some disorders are introduced. The last and the first layer of $\mathrm{PC}_{1}$ and $\mathrm{PC}_{2}\left(d_{2 \mathrm{SiO}_{2}}\right)$ on the left and right sides respectively are deformed by $\delta d= \pm 0.05 a_{2}$ with changing the refractive index of the middle $\mathrm{SiO}_{2}$ in the chirped PC by $\delta n= \pm 0.01$. Figure $6 \mathrm{C}$ shows the formed trivial rainbow trapping with disordering. Two points are observed clearly between the two cases; some localized modes occurred at the same wavelengths, and others slightly shifted. However, the case of $1,493.69 \mathrm{~nm}$ is localized at a different position without defects.

\section{REFERENCES}

1. Tsakmakidis KL, Boardman AD, Hess O. 'Trapped Rainbow' Storage of Light in Metamaterials. Nature (2007) 450(7168):397-401. doi:10.1038/nature06285

2. Hu H, Ji D, Zeng X, Liu K, Gan Q. Rainbow Trapping in Hyperbolic Metamaterial Waveguide. Sci Rep (2013) 3(1):1249. doi:10.1038/srep01249

3. Xu Z, Shi J, Davis RJ, Yin X, Sievenpiper DF. Rainbow Trapping with Long Oscillation Lifetimes in Gradient Magnetoinductive Metasurfaces. Phys Rev Appl (2019) 12(2):024043. doi:10.1103/PhysRevApplied.12.024043

4. Gan Q, Ding YJ, Bartoli FJ. "Rainbow" Trapping and Releasing at Telecommunication Wavelengths. Phys Rev Lett (2009) 102(5):056801. doi:10.1103/PhysRevLett.102.056801

5. Chen L, Wang GP, Gan Q, Bartoli FJ. Rainbow Trapping and Releasing by Chirped Plasmonic Waveguides at Visible Frequencies. Appl Phys Lett (2010) 97(15):153115. doi:10.1063/1.3502487

6. Schuller JA, Barnard ES, Cai W, Jun YC, White JS, Brongersma ML. Plasmonics for Extreme Light Concentration and Manipulation. Nat Mater (2010) 9(3):193-204. doi:10.1038/nmat2630

\section{CONCLUSIONS}

In conclusion, a unidirectional and bidirectional rainbow trapping have been investigated. The main idea is based on trapping a chirped PC as a sandwich between two edge states. At the same time, the two edge states work as a cavity to trap the light inside the chirped structure and can be propagated in both directions. Consequently, different frequencies from the wave packet segregate at different positions in both directions. Moreover, the propagation properties are validated using FDTD by measuring the electric field intensity amplitude. In addition, we confirmed that the formed rainbow is robust and topologically protected even in the presence of disorders. Bidirectional rainbow trapping will open a feasible way for numerous applications, e.g., multichannel wavelength demultiplexers, bidirectional optical filter, and bidirectional laser.

\section{DATA AVAILABILITY STATEMENT}

The original contributions presented in the study are included in the article/Supplementary Material, further inquiries can be directed to the corresponding author.

\section{AUTHOR CONTRIBUTIONS}

SE conceived the idea, performed the numerical simulations, and wrote the draft of the manuscript. CL checked the simulation results and revised the manuscript. CL supervised the project.

\section{FUNDING}

National Natural Science Foundation of China (91850117, 11654003); Beijing Institute of Technology Research Fund Program for Young Scholars.

7. Chaplain GJ, De Ponti JM, Aguzzi G, Colombi A, Craster RV. Topological Rainbow Trapping for Elastic Energy Harvesting in Graded Su-SchriefferHeeger Systems. Phys Rev Appl (2020) 14(5):054035. doi:10.1103/ PhysRevApplied.14.054035

8. Ungureanu B, Makwana MP, Craster RV, Guenneau S. Localizing Elastic Edge Waves via the Topological Rainbow Effect. Phys Rev Appl (2021) 15(1):014057. doi:10.1103/PhysRevApplied.15.014057

9. Shen Y, Fu J, Yu G. Rainbow Trapping in One-Dimensional Chirped Photonic Crystals Composed of Alternating Dielectric Slabs. Phys Lett A (2011) 375(43): 3801-3. doi:10.1016/j.physleta.2011.08.023

10. Lu C, Wang C, Xiao M, Zhang ZQ, Chan CT. Topological Rainbow Concentrator Based on Synthetic Dimension. Phys Rev Lett (2021) 126(11): 113902. doi:10.1103/PhysRevLett.126.113902

11. Zhang H, Qian L, Wang C, Lu C., Liu Y, Chen J, et al. Topological Rainbow Based on Graded Topological Photonic Crystals. Opt Lett (2021) 46(6): 1237-40. doi:10.1364/OL.419271

12. Hayran Z, Kurt H, Staliunas K. Rainbow Trapping in a Chirped ThreeDimensional Photonic crystal. Sci Rep (2017) 7(1):3046. doi:10.1038/ s41598-017-03454-w 
13. Elshahat S, Abood I, Esmail MSM, Ouyang Z, Lu C. One-Dimensional Topological Photonic Crystal Mirror Heterostructure for Sensing. Nanomaterials (2021) 11(8):1940. doi:10.3390/nano11081940

14. Dal Lago V, Atala M, Foa Torres LEF. Floquet Topological Transitions in a Driven One-Dimensional Topological Insulator. Phys Rev A (2015) 92(2): 023624. doi:10.1103/PhysRevA.92.023624

15. Kurt H, Yilmaz D. Rainbow Trapping Using Chirped All-Dielectric Periodic Structures. Appl Phys B (2013) 110(3):411-7. doi:10.1007/s00340-012-5270-8

Conflict of Interest: The authors declare that the research was conducted in the absence of any commercial or financial relationships that could be construed as a potential conflict of interest.
Publisher's Note: All claims expressed in this article are solely those of the authors and do not necessarily represent those of their affiliated organizations, or those of the publisher, the editors and the reviewers. Any product that may be evaluated in this article, orclaim that may be made by its manufacturer, is not guaranteed or endorsed by the publisher.

Copyright $\odot 2022$ Elshahat and Lu. This is an open-access article distributed under the terms of the Creative Commons Attribution License (CC BY). The use, distribution or reproduction in other forums is permitted, provided the original author(s) and the copyright owner(s) are credited and that the original publication in this journal is cited, in accordance with accepted academic practice. No use, distribution or reproduction is permitted which does not comply with these terms. 